\title{
Sífilis congênita em cinco regiões de saúde do estado de Santa Catarina no período 2010 a 2019
}

\author{
Congenital Syphilis in five health \\ regions in the state of Santa Catarina \\ in the period 2010 to 2019
}

\section{Soraia Laísa Nass ${ }^{1}$ \\ Vitória Chiapetti dos Santos ${ }^{2}$ (1) Lidiane Ferreira Schultz ${ }^{3}$ (1) Tadiana Maria Alves Moreira ${ }^{4}$ (1)}

\begin{abstract}
1,2,4Faculdade IELUSC (Joinville). Santa Catarina, Brasil. so.laisa@hotmail.com, vichiapetti@hotmail.com, tadiana.moreira@ielusc.br
\end{abstract} ${ }^{3}$ Autora para correspondência. Faculdade IELUSC (Joinville). Santa Catarina, Brasil. lidiane.schultz@ielusc.br

RESUMO I OBJETIVO: Conhecer o perfil de morbidade e mortalidade da sífilis congênita em cinco Regiões de Saúde de Santa Catarina no período de 2010 a 2019. MÉTODOS E MATERIAIS: Estudo ecológico descritivo, retrospectivo, com abordagem quantitativa, realizado em Santa Catarina, em cinco Regiões de Saúde de um total de 16 regiões selecionadas segundo a distribuição geográfica do Plano Diretor de Regionalização/2012. Utilizou-se dados secundários do Sistema de Informação de Agravos de Notificação e populacionais do Departamento de Informática do Sistema Único de Saúde. Os dados coletados foram organizados, tabulados e descritos em frequências absolutas e relativas para identificação das caraterísticas clínicas do recém-nascido e o perfil epidemiológico a partir da construção de indicadores de morbidade e mortalidade. RESULTADOS: As maiores incidências ocorreram nas Regiões de Saúde Nordeste e Serra Catarinense, ainda que essas estejam abaixo de 10 casos/1000 Nascidos Vivos. As taxas de mortalidade nas Regiões de Saúde são baixas. As mais elevadas estão na Região de Saúde Oeste (53,34 casos/100.000 NV) e Serra Catarinense (25,11 casos/100.000 NV). CONCLUSÃO: Com este estudo foi possível descrever o perfil da morbidade e mortalidade da sífilis congênita em cinco Regiões de Saúde de Santa Catarina, no período de 2010 a 2019, indicando um problema importante de saúde pública em Santa Catarina.

DESCRITORES: Sífilis congênita. Morbidade. Mortalidade. Epidemiologia.

\begin{abstract}
OBJECTIVE: To know the morbidity and mortality profile of congenital syphilis in five Health Regions of Santa Catarina from 2010 to 2019. METHODS AND MATERIALS: A descriptive, retrospective ecological study with a quantitative approach carried out in Santa Catarina, in five Health Regions from a total of 16 regions, selected according to the geographic distribution of the Regionalization Master Plan/2012. Secondary data from the Information System on Notifiable and Population Diseases of the Informatics Department of the Unified Health System were used. The collected data were organized, tabulated, and described in absolute and relative frequencies to identify the clinical characteristics of the newborn and the epidemiological profile based on the construction of morbidity and mortality indicators. RESULTS: The highest incidence occurred in the Northeast and Serra Catarinense Health Regions, although these are below 10 cases/1000 Live Births. Mortality rates in the Health Regions are low. The highest rates are in the Western Health Region (53.34 cases/100,000 LB) and Serra Catarinense (25.11 cases/100,000 LB). CONCLUSION: With this study, it was possible to describe the morbidity and mortality profile of congenital syphilis in five Health Regions of Santa Catarina in the period from 2010 to 2019 , and this disease remains an important public health problem in Santa Catarina.
\end{abstract}

KEYWORDS: Congenital syphilis. Morbidity. Mortality. Epidemiology. 


\section{Introdução}

A sífilis congênita, doença milenar, continua sendo um problema de saúde pública devido a sua alta transmissão vertical. É uma infecção bacteriana sistêmica, causada pelo Treponema pallidum, transmitida também por via sexual ${ }^{1}$, mas de fácil diagnóstico e com tratamento gratuito.?

A transmissão vertical pode trazer consequências imediata ou tardia no bebê, como aborto, natimorto, parto pré-termo, morte neonatal e manifestações congênitas.? Essa doença pode ser evitada através do acompanhamento das mulheres com sífilis e da realização e tratamento adequado durante o pré-natal. 3 Outros fatores também estão associados ao risco aumentado de infecção por sífilis durante a gravidez como disparidades étnicas, baixo nível socioeconômico, pouca escolaridade, práticas sexuais inseguras, acesso limitado ou falha na triagem e atendimentos nos serviços de saúde. ${ }^{4,5}$

Sífilis é uma doença de notificação compulsória e deve ser realizada por todos os profissionais da rede de saúde pública e privada para investigação do caso e confirmação ou não do mesmo, no Sistema de Informação de Agravos de Notificação - SINAN. ${ }^{2}$

De acordo com a Organização Mundial de Saúde, em 2016, a estimativa de casos da doença no mundo era

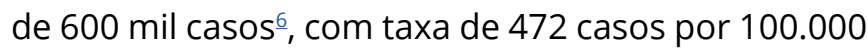
nascidos vivos, resultando em mais de 200.000 natimortos e mortes neonatais precoces. ${ }^{?}$

Em 2017, nos Estados Unidos, a taxa nacional de sífilis congênita foi de 23,3 casos por 100.000 nascidos vivos, a maior taxa desde 1997. $\frac{5 \mathrm{Em}}{2018}$, em relação a 2013 , houve um aumento de $261 \%$ no país. ${ }^{8}$ Essas taxas foram 6,4 vezes e 3,3 vezes maiores entre recém-nascidos de mães negras e hispânicas quando comparadas com as mães brancas. .5

No Brasil, de acordo com o Boletim Epidemiológico do Ministério da Saúde, verificou-se um aumento substancial da taxa de sífilis congênita nas últimas duas décadas 2 , sendo notificados, no período entre 1998 e 2019, 414.891 casos em menores de um ano de idade no país; 8,5\% (18.119) na Região Sul.?
Em 2018, foram confirmados $25.889(98,4 \%)$ casos de sífilis congênita em neonatos no Brasil; 25.456 $(96,8 \%)$ foram diagnosticados na primeira semana de vida e, desses casos confirmados, 93,6\% foram classificados como de sífilis congênita recente. ${ }^{2}$ A Taxa de incidência da sífilis congênita foi de 8,2/100.000 nascidos vivos. Onze estados brasileiros apresentaram uma taxa de incidência de maior que 9,0/1.000 nascidos vivos no ano em questão.?

Em Santa Catarina, em 2018, foram 680 casos novos de sífilis congênita com uma taxa de incidência de $6,8 / 1.000$ nascidos vivos e 28 óbitos (taxa de mortalidade de 28,1 casos/100 mil nascidos vivos. ${ }^{8}$

O aumento de sífilis congênita em Santa Catarina na última década, associado a limitados estudos sobre o perfil de morbi/mortalidade e da distribuição espacial da doença nas 16 Regiões de Saúde que compõem o Plano Diretor de Regionalização, favoreceu a escolha desta temática de pesquisa.

Estudos que descrevam sobre o perfil de morbi/mortalidade podem auxiliar na tomada de decisões e na programação de ações em saúde, auxiliando nas políticas, programas e na ampliação do conhecimento de profissionais de saúde para monitoração, prevenção e assistência a população.

Assim, a pergunta que norteou este estudo foi: qual o perfil de morbi/mortalidadade da sífilis congênita nas Regiões de Saúde em Santa Catarina no período 2010 a 2019? O objetivo deste estudo foi conhecer o perfil de morbidade e mortalidade da sífilis congênita em cinco Regiões de Saúde do Estado de Santa Catarina, no período 2010 a 2019.

\section{Método}

Estudo ecológico, descritivo, retrospectivo, com abordagem quantitativa, que busca informar sobre a distribuição da ocorrência de sífilis congênita nas crianças menores de um ano de idade, no período 2010 a 2019. 
A proposta inicial deste estudo era analisar o perfil epidemiológico da sífilis congênita de todo o estado de Santa Catarina, segundo a divisão do Plano Diretor de Regionalização - PDR, que é composto por 16 Regiões de Saúde - RS. 9 Entretanto, por ser um estudo ecológico, longitudinal, de uma série histórica de 10 anos, o tempo disponível para a coleta e análise dos dados tornou-se um limitador para a realização do cálculo de cada indicador epidemiológico proposto para as 16 RS. Assim, foi definido por trabalhar cinco Regiões de Saúde (RS) de Santa Catarina: Foz do Rio Itajaí, Serra Catarinense, Grande Florianópolis, Nordeste e Oeste, localizadas, geograficamente, em pontos distintos do mapa geográfico.

A pesquisa foi realizada no estado de Santa Catarina, tendo como base as Regiões de Saúde criadas no Plano Diretor de Regionalização (2012), no cumprimento do Contrato Organizativo de Ações Públicas, da Resolução 7.508/201.?

Os participantes deste estudo foram todas as crianças com até um ano de idade que tiveram o diagnóstico confirmado de sífilis congênita, no período 2010 a 2019, de mães que residem em um dos municípios nas cinco Regiões de Saúde escolhidas.

Os dados da pesquisa foram coletados no mês de juIho de 2020, no Sistema de Informação de Agravos de Notificação (SINAN) e no Sistema de Informação sobre Mortalidade (SIM), disponíveis no site da Diretoria de Vigilância Epidemiologia, da Secretaria de Saúde de Santa Catarina. Já os dados populacionais foram obtidos no site do Departamento de Informática do Sistema Único de Saúde - DATASUS. Estes foram organizados segundo a variável independente, ano de notificação/confirmação. Já como variáveis dependentes, foram selecionados e analisados o sexo, realização do teste não treponêmico (reagente), recémnascido sintomático/assintomático e diagnóstico recente, constantes da ficha do SINAN.

Os dados são inicialmente tabulados, organizados, em uma planilha do programa Microsoft Office Exce/ ${ }^{\circledR}$ 2013. Após, foram calculadas as frequências absolutas e relativas das características clínicas dos recém-nascidos. Em relação às características clínicas, foram trabalhados os dados clínicos de recém-nascidos que tiveram ao nascer o teste não treponêmico reagente, recém-nascidos com diagnóstico de Sífilis Congênita recente e recém-nascidos sintomáticos.
Para se conhecer o perfil epidemiológico da doença nas Regiões de Saúde selecionadas, primeiramente foram calculadas as taxas de incidência específica para cada ano da série histórica, de cada Região de Saúde. Também calculadas as taxas específicas de incidência, segundo o sexo.

Para melhor compreender a morbidade, decidiu-se por apresentar a taxa de incidência específica e as taxas de incidência por sexos de sífilis congênita das cinco Regiões de Saúde, a partir das médias dos indicadores taxa de incidência específica, taxa de incidência de sífilis congênita segundo o sexo (masculino e feminino, respectivamente) da série histórica, que permite comparar de maneira mais clara, os resultados das médias dos indicadores epidemiológicos das cinco regiões e, assim, compreender os resultados pelas suas comparações.

Em relação à análise da mortalidade, adotou-se a constante de multiplicação para cada 100 mil nascidos vivos, uma vez que o número de óbitos por sífilis congênita é baixo nas regiões selecionadas.

Todos os preceitos éticos foram cumpridos em todas as etapas da pesquisa, de acordo com Resolução $n$ -466/2012, do Ministério da Saude.10 O Projeto de pesquisa foi aprovado pelo Comitê de Ética em Pesquisa com Parecer $n^{\circ} 4.030 .171$, de 15 de maio de 2020.

\section{Resultados}

Os resultados dos indicadores construídos são apresentados e foram organizados segundo as características clínicas dos recém-nascidos, o perfil de morbidade e perfil de mortalidade.

Em relação às caraterísticas clínicas, a Tabela 1 mostra que a Região de Saúde Nordeste apresentou maior proporção de recém-nascidos com teste não treponêmico reagente após o nascimento (92,65\%), e a Região Oeste, a menor proporção média (62,32\%). A maioria dos recém-nascidos é diagnosticada com sífilis congênita recente, cuja proporção variou de 89,20\% (Foz do Rio Itajaí) a 99,23\% (Oeste). No nascimento, a incidência de casos de recém-nascidos que apresentam sintomas da doença nas Regiões de Saúde Nordeste (5,51\%) e Serra Catarinense (20,94\%), conforme apresentado na Tabela 1. 
Tabela 1. Proporção média das características clínicas de recém-nascidos com sífilis congênita segundo cinco Regiões de Saúde. Santa Catarina $2010-2019$

\begin{tabular}{|c|c|c|c|c|c|}
\hline \multirow[b]{2}{*}{ Indicadores } & \multicolumn{5}{|c|}{ Regiões de Saúde } \\
\hline & Nordeste & $\begin{array}{l}\text { Foz do Rio } \\
\text { Itajaí }\end{array}$ & $\begin{array}{l}\text { Grande } \\
\text { Fpolis. }\end{array}$ & $\begin{array}{c}\text { Serra } \\
\text { Catarinense }\end{array}$ & Oeste \\
\hline $\begin{array}{l}\text { Proporção de casos com teste } \\
\text { não trep. Reagente }\end{array}$ & $92,65 \%$ & $87,81 \%$ & $91,59 \%$ & $90,61 \%$ & $62,32 \%$ \\
\hline $\begin{array}{l}\text { Proporção de casos com } \\
\text { diagnóstico de sífilis congênita } \\
\text { recente }\end{array}$ & $90,22 \%$ & $89,20 \%$ & $91,00 \%$ & $92,56 \%$ & $99,23 \%$ \\
\hline $\begin{array}{l}\text { Proporção de casos RN } \\
\text { sintomáticos }\end{array}$ & $5,51 \%$ & $8,92 \%$ & $9,81 \%$ & $20,94 \%$ & $15,33 \%$ \\
\hline
\end{tabular}

Na Figura 1, referente a categoria Perfil de Morbidade, verifica-se que a taxa de incidência de sífilis congênita do estado de Santa Catarina nos anos de 2010 a 2018 apresentou uma discreta ascendência, sendo mais alta no ano de 2018, com 6,40 casos/1.000 NV. Nas taxas de incidência, segundo as Regiões de Saúde, constata-se que em todas houve um aumento das taxas de incidência a partir de 2014. Entretanto, a Região de Saúde Serra Catarinense apresenta uma ascendência abrupta da curva, atingindo em 2019 uma taxa de 36,58 casos novos em cada 1.000 nascidos vivos, totalmente acima das demais regiões analisadas.

Figura 1. Série histórica da taxa de incidência de sífilis congênita por 1.000 nascidos vivos segundo cinco Regiões de Saúde. Santa Catarina. $2010-2019$

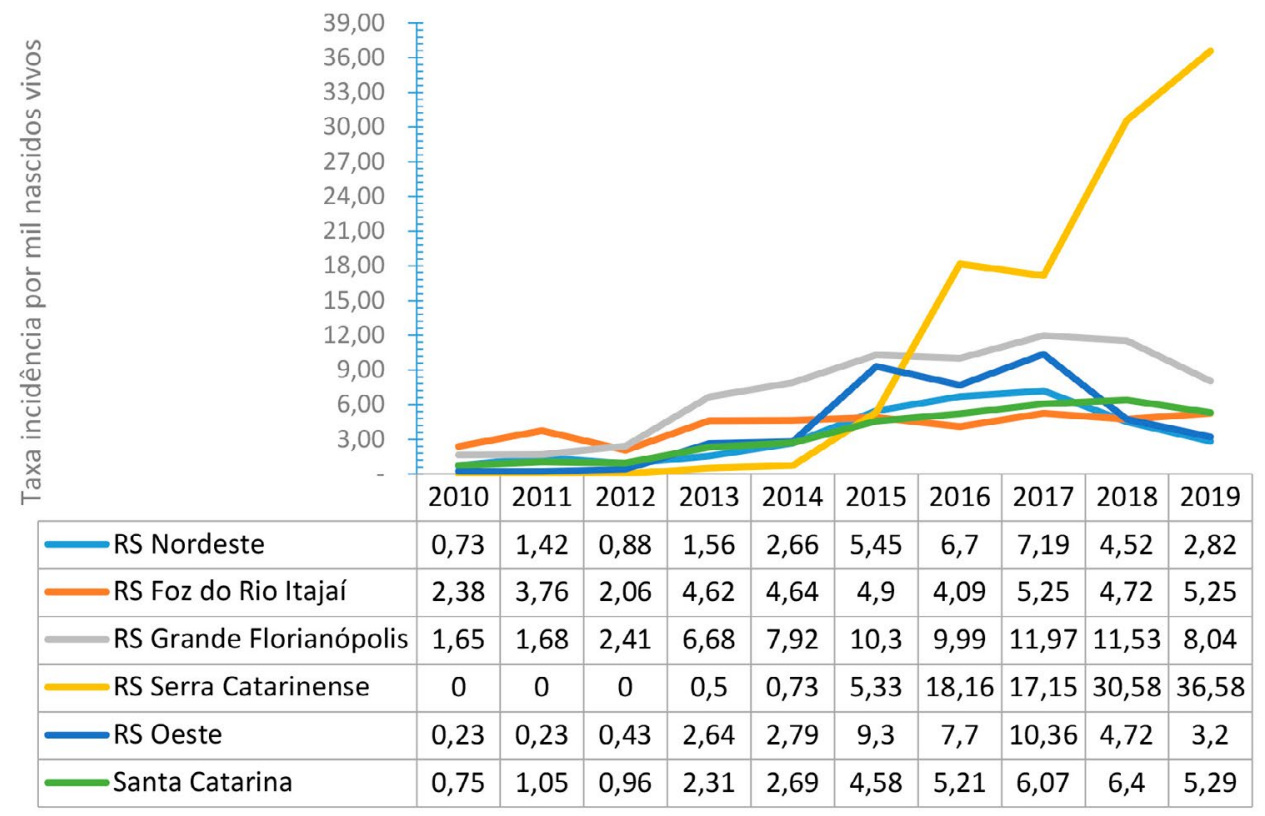

Fonte: Dive/SES-SC 
A tabela 2 traz as taxas médias de incidência específica e taxa média de incidência segundo sexo, calculadas por 1.000 nascidos vivos. A Serra Catarinense possui uma taxa média de incidência de sífilis congênita de 15,57 casos/1.000 NV, caracterizando a maior taxa entre as cinco Regiões de Saúde analisadas, sendo que a menor taxa, de 3,36 casos/1.000 NV, ocorre na Região de Saúde Nordeste.

Os resultados das taxas médias de incidências por sexos mostram o mesmo comportamento da taxa média de incidência total. A Região de Saúde Serra Catarinense novamente apresentou a maior taxa média, 16,18/1.000 NV no sexo feminino. As menores taxas, tanto na média total como na taxa média no sexo feminino, ocorreu na RS Nordeste, 3,36/1.000 NV e 3,05/1.000 NV femininos, respectivamente. Os resultados da taxa média de incidência no sexo masculino mostram que a menor taxa foi na Região Nordeste (2,97 casos/1.000 NV), enquanto a maior taxa se deu também na Serra Catarinense, com 13,04/1.000 NV meninos, mantendo o mesmo padrão de morbidade (Tabela 2).

Tabela 2. Taxa média de incidência e por sexo de sífilis congênita por 1.000 nascidos vivos segundo cinco Regiões de Saúde. Santa Catarina $2010-2019$

\begin{tabular}{lccccc}
\hline Indicadores & Nordeste & $\begin{array}{c}\text { Foz do Rio } \\
\text { Itajaí }\end{array}$ & $\begin{array}{c}\text { Grande } \\
\text { Fpolis. }\end{array}$ & $\begin{array}{c}\text { Serra } \\
\text { Catarinense }\end{array}$ & Oeste \\
\hline $\begin{array}{l}\text { Taxa média de I } \\
\text { específica total }\end{array}$ & 3,36 & 4,17 & 7,22 & 15,57 & 4,16 \\
$\begin{array}{l}\text { Taxa de média I do } \\
\text { Sexo M }\end{array}$ & 2,97 & 3,53 & 6,49 & 13,04 & 4,35 \\
$\begin{array}{l}\text { Taxa de média I do } \\
\text { Sexo F }\end{array}$ & 3,05 & 4,30 & 7,05 & 16,18 & 3,96 \\
\hline $\begin{array}{l}\text { Fonte: DIVE/SC } \\
\text { T. }\end{array}$ & & & &
\end{tabular}

Outro critério de análise deste estudo refere-se ao perfil de mortalidade cujos indicadores utilizados permitem avaliar a gravidade da doença. Em relação à taxa de mortalidade específica por sífilis congênita e a taxa segundo os sexos no estado de Santa Catarina, é possível verificar que esses índices são baixos.

Nos resultados apresentados na figura 2, verifica-se uma estabilidade na taxa de mortalidade de aproximadamente 1,18 casos/100.000 NV em Santa Catarina nos anos de 2010 a 2012, com maior taxa em 2015 (12,22 casos/100.000 NV), reduzindo para 6,15 casos para cada 100 mil nascidos vivos em 2019.

Nos resultados das cinco Regiões de Saúde, observa-se que a mortalidade por sífilis congênita é baixa, com oscilações nas curvas das Regiões de Saúde analisadas. As Regiões Oeste e Serra Catarinense apresentam um aumento na taxa de mortalidade nos anos de 2012 (21,54/100.000 NV) e 2013 (25,11/100.000 NV), respectivamente. A Região Oeste mantém uma taxa de mortalidade próxima a 20 óbitos para cada 100 mil nascidos vivos no período 2015 a 2017, mas, em 2019, esta aumenta para 53,34/100.000 NV. Comportamento semelhante observa-se na Região Serra Catarinense no mesmo período 2015 a 217, com taxas de mortalidade próximas a 24 óbitos por 100 mil nascidos vivos. Já na Região Foz do Rio Itajaí, no período 2014 a 2016 e no ano de 2019, as taxas de mortalidade estão próximas a 10/1000.000 NV. 


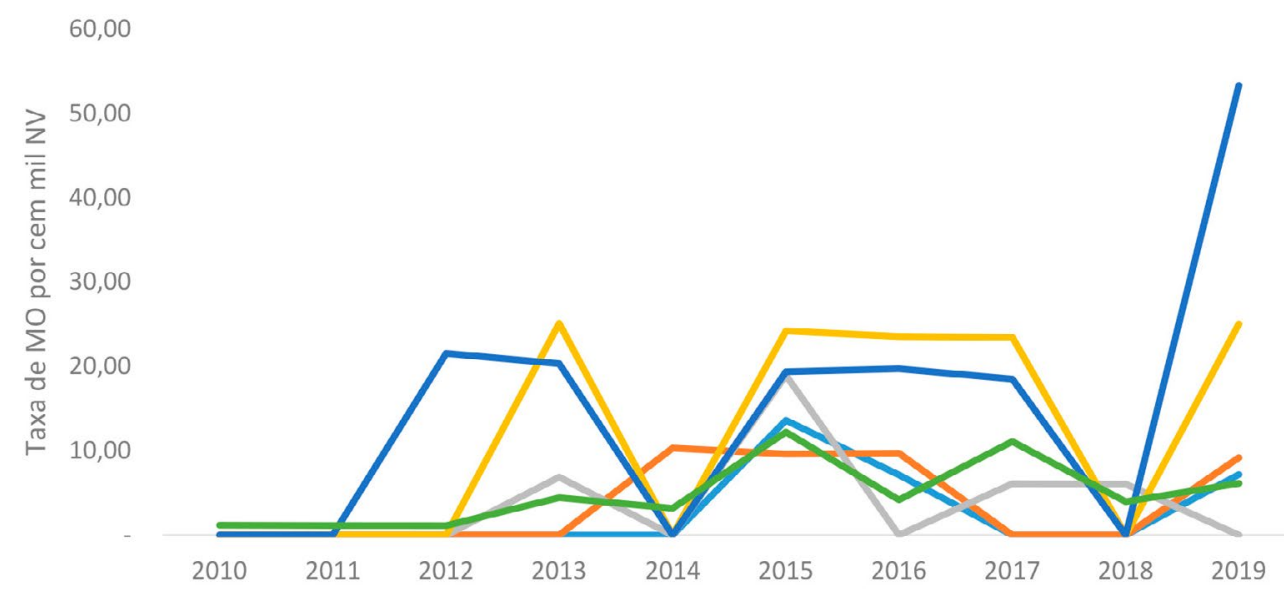

Fonte: Dive/SES-SC

A tabela 3 apresenta as taxas médias de mortalidade específica da sífilis congênita e também estratifica segundo o sexo das cinco Regiões de Saúde selecionadas para este estudo. Observa-se que as taxas médias de mortalidade, como esperado, são mais altas nas Região de Saúde Serra Catarinense (24,29/100.000NV) e Oeste $(25,47 / 100.000 \mathrm{NV})$. Quando estratificadas segundo o sexo, as maiores taxas são observadas no sexo masculino com 47,67/100.000 NV, na RS Serra Catarinense. Já no sexo feminino, a Região Oeste apresenta 26,73/100.000 $\mathrm{NV}$; a maior taxa média das regiões analisadas.

Tabela 3. Taxas médias de mortalidade específica e por sexo de sífilis congênita segundo cinco Regiões de Saúde. Santa Catarina $2010-2019$

\begin{tabular}{lcccc}
\hline & \multicolumn{3}{c}{ Regiões } \\
\cline { 2 - 5 } Indicadores & Nordeste & $\begin{array}{c}\text { Foz do Rio } \\
\text { Itajaí }\end{array}$ & $\begin{array}{c}\text { Grande } \\
\text { Fpolis. }\end{array}$ & $\begin{array}{c}\text { Serra } \\
\text { Catarinense }\end{array}$ \\
\hline $\begin{array}{l}\text { Taxa média de Mortalidade } \\
\text { específica total }\end{array}$ & 9,31 & 9,72 & 9,49 & 24,29 \\
$\begin{array}{l}\text { Taxa média de Mortalidade do } \\
\text { sexo masculino }\end{array}$ & 13,6 & 4,65 & 6,03 & 25,47 \\
$\begin{array}{l}\text { Taxa média de Mortalidade do } \\
\text { sexo feminino }\end{array}$ & 4,93 & 14,97 & 13,13 & 24,74 \\
\hline *100.000 Nascidos vivos & Fonte: DIVE/SC & & & 26,73 \\
\hline
\end{tabular}

Finalmente, buscou-se conhecer a taxa de letalidade por sífilis congênita, a partir da construção desse indicador para cada uma das cinco Regiões de Saúde analisadas. Na tabela 4, verifica-se que as taxas de letalidade variam de 0,51 óbitos em cada 100 casos de sífilis, no ano de 2017, na Região de Saúde Grande Florianópolis, para 50,00 óbitos nas Regiões Oeste (2012) e Serra Catarinense (2013), respectivamente. No entanto, cabe esclarecer que essas altas taxas de letalidade são decorrentes do baixo número de casos confirmados $(n=2)$ em cada uma das regiões. As demais Regiões de Saúde apresentaram taxas baixas, que varia de 0,51 na Grande Florianópolis até 16,7 óbitos na Região Oeste, em 2019. 
Tabela 4. Taxas de letalidade da Sífilis congênita segundo cinco Regiões de Saúde. Santa Catarina. 2010-2019

\begin{tabular}{|c|c|c|c|c|c|c|c|c|c|c|}
\hline Regiões de Saúde & 2010 & 2011 & 2012 & 2013 & 2014 & 2015 & 2016 & 2017 & 2018 & 2019 \\
\hline RS Nordeste & - & - & - & - & - & 2,50 & 1,06 & - & - & 2,86 \\
\hline RS Foz do Rio Itajaí & - & - & - & - & 2,22 & 1,96 & 2,38 & - & - & 1,75 \\
\hline \multicolumn{11}{|l|}{ RS Grande } \\
\hline Florianópolis & - & - & - & 1,03 & - & 1,84 & - & 0,51 & 0,52 & - \\
\hline RS Serra Catarinense & - & - & - & 50,00 & - & 4,55 & 1,30 & 1,37 & - & 0,68 \\
\hline RS Oeste & - & - & 50,00 & 7,69 & - & 2,08 & 2,56 & 1,79 & - & 16,7 \\
\hline
\end{tabular}

\section{Discussão}

O controle de doenças infectocontagiosas como a sífilis é responsabilidade da atenção primária a saúde, na adoção das medidas de controle direcionadas não apenas à gestante, mas também a seu parceiro, para que através de tratamento eficaz possa quebrar a cadeia de transmissão da doença, evitando, assim, a ocorrência de uma doença evitável ao recém-nascidos, a sífilis congênita.

Ainda que medidas de controle estejam definidas nacionalmente para o controle da sífilis congênita, este estudo mostra que nas cinco Regiões de Saúde catarinenses o comportamento da doença se dá de maneira diferenciadas.

A análise das características clínicas dos recém-nascidos deste estudo mostra que em relação ao diagnóstico da sífilis recente, o percentual nas cinco Regiões de Saúde analisadas foi superior a 90\%. No estudo realizado em Recife, entre 2011-2015, foi identificado que 47,36\% dos resultados dos testes sanguíneos não treponemos em sangue periférico de recém-nascidos deram reagentes, sendo que 5,26\% apresentavam sintomatologia. ${ }^{11} \mathrm{Em}$ outra pesquisa em um município de São Paulo, $61 \%$ dos casos foram reagentes e $20 \%$ não reagentes. E as alterações ósseas, essas estavam presentes em $14 \%$ das notificações. $\frac{12}{2}$

Na Região Sul do Brasil, entre 2001 e 2009, 86,9\% dos casos de sífilis congênita foram diagnosticadas como recentes, proporção próxima às encontradas também foram encontrados em outro estudo brasileiro. ${ }^{13}$

A realização do pré-natal foi de $79,29 \%$ das gestantes, conforme recomendações do Ministério da Saúde..$^{14}$ Gestantes que obtiveram o diagnóstico de sífilis durante as consultas de pré-natal foi de $60,01 \%$, porém $55,05 \%$ dos tratamentos foram inadequados. 12 Outro estudo mostrou que apesar de $96,6 \%$ das gestantes realizarem o pré-natal adequadamente, $40 \%$ delas obtiveram o diagnóstico de sífilis no momento do parto, comprometendo a realização do tratamento, levando ao aumento dos casos de sífilis congênita. ${ }^{15}$

O tratamento inadequado das gestantes e parceiros é um grande desafio e favorece o aumento e ocorrência da sífilis congênita, sendo descrito em alguns estudos ${ }^{12,13,16}$ e indicando a necessidade de melhorias. Concomitantemente, o Ministério da Saúde aponta para a importância também de o diagnóstico de crianças com sífilis congênita ser realizado precocemente. $\underline{2}$

Em relação à taxa de incidência de sífilis congênita, o estado de Santa Catarina apresentou o menor valor no ano de 2010, com uma taxa de incidência de 0,75/1.000 nascidos vivos, que não alcança a meta preconizada pela OPAS/OMS de 0,5 casos/1.000 NV17 na série analisada no presente estudo. O comportamento da doença, nos anos de 2010 a 2018, não apresentou uma grande modificação, com a maior taxa de incidência em 2018, com 6,40 casos/1.000 NV. 
Um estudo realizado nas capitais brasileiras descreveu 44.056 casos de sífilis congênita em menores de um ano entre 2009 e 2016. $\frac{18}{\text { O }}$ maior índice de sífilis congênita ocorreu em 2016 em Porto Alegre (31,07 /1.000 nascidos vivos). 18 As capitais nordestinas obtiveram destaque quanto às taxas mais elevadas, em especial a capital Recife (23,67 / 1.000 nascidos vivos). .18

Nestes estudos verifica-se que as taxas ultrapassam a média 0,5/1000 NV, adotada pelo Ministério da Saúde, de acordo com as recomendações da Organização Pan-americana de Saúde. ${ }^{17}$

No que diz respeito às taxas médias de incidências por sexos com exceção da Região de Saúde Serra Catarinense, as demais regiões analisadas mantiveram uma média de sete casos para cada mil nascidos vivos. Estudo sobre análise dos casos de sífilis congênita realizado em Sobral, Ceará mostrou que do total de nove bebês que nasceram com sífilis, oito eram do sexo feminino. $\frac{19}{}$

O acompanhamento da gestante no pré-natal e tratamento realizado na maternidade a partir do diagnosticado realizado no recém-nascido no momento do nascimento são fundamentais.

Quanto ao perfil de morbidade, duas Regiões de Saúde destacam-se com altas taxas de incidência, ambas localizadas distantes dos centros urbanos, o que pode dificultar o acesso a um diagnóstico recente no momento do parto e ao tratamento oportuno, que exige a permanência do recém-nascido internado na maternidade. Soma-se ainda a possibilidade da realização de parto domiciliar em regiões rurais, as duas regiões de saúde mais endêmicas de nosso estudo. Outros estudos confirmam sobre condições sociais desfavoráveis, vulnerabilidade das gestantes, baixa escolaridade, renda insuficiente e limitado acesso aos serviços de saúde relacionados a morbidade por sífilis congênita. $\frac{12,13,20}{2}$

O perfil de mortalidade por sífilis congênita expressa a gravidade que a doença possa apresentar para recém-nascidos e crianças até dois anos de idade, através da taxa de mortalidade específica e da taxa de mortalidade específica segundos os sexos. Cabe mencionar resultados bastante baixos desses indicadores nesta pesquisa, que sugerem efetividade das medidas de controle adotadas no estado de Santa Catarina, principalmente no pré-natal da gestante, assim como nas medidas voltadas aos recém-nascidos com diagnóstico recente de sífilis congênita, tratamento imediato após o parto e durante o processo de internado na maternidade.

Quando se compara a taxa média de mortalidade específica e as taxas médias de mortalidade específicas das Regiões de Saúde analisadas com os resultados no Brasil, verifica-se grandes disparidades nos achados encontrados neste estudo. Nas Regiões de Saúde analisadas, principalmente a Serra Catarinense e Oeste, essas taxas são muito altas, quase 24 vezes maiores que no Brasil. No Brasil, nos últimos dez anos, os óbitos por sífilis congênita tiveram uma taxa de 1,9/100.000 NV em 2008, chegando a 8,2/100.000 NV em 2018. ${ }^{2}$

Casos de sífilis congênita, que pode ter como uma das consequências a mortalidade perinatal, podem estar relacionado ao tratamento em dosagem incorrenta ou não realização do tratamento da sífilis materna. .11 $^{21}$

Para a redução da transmissãoo da sífilis congênita torna-se necessário a adoção de medidas de controle, que consistem na melhoria no acesso as unidades básicas de saúde e na qualidade da assistência pré-natal; no aumento dos testes de screening na primeira consulta pré-natal; na adequação do tratamento apropriado para as mulheres e parceiros infectados e na expansão dos programas de intervenção voltados para os grupos de alto risco. 22

Garantir atenção de qualidade durante o parto e puerpério, resguardar ao recém-nascido o direito de nascer com segurança e crescer de forma saudável são princípios que precisam ser implementados a partir dos programas e políticas públicas na assistência materno-infantil. $\underline{23}$

Para a modificação do perfil de morbi/mortalidade por sífilis congênita outro fator importante consiste na maior participação e melhor capacitação das equipes da atenção básica, as quais são de extrema importância por estarem trabalhando na linha de frente com essas famílias, colaborando, assim, para a promoção da saúde, visando reduzir essa doença para o alcance da meta preconizada. ${ }^{24}$ 


\section{Conclusões}

A realização deste estudo permitiu conhecer o perfil de morbi/mortalidade da sífilis congênita e identificar as diferenças em cinco Regiões de Saúde do estado de Santa Catarina, no período 2010 a 2019. A não realização sistemática para o diagnóstico da sífilis na gestação, pode ter contribuído nos últimos anos do estudo para o aumento do número de recém-nascidos com sífilis congênita. Sendo os diagnósticos confirmados em maior prevalência como assintomáticos.

Quanto ao perfil de morbidade, duas Regiões de Saúde destacam-se com altas taxas de incidência, ambas localizadas distantes dos centros urbanos. Já no perfil de mortalidade, se verifica no período de 2010 a 2017 a manutenção de baixas taxas de mortalidade e a não ocorrência de óbitos em diversos anos. No entanto, nos anos de 2018 e 2019 identifica-se uma mudança no perfil de mortalidade com maiores taxas na Região Serra Catarinense (2019) e Região Oeste em todo o período analisado.

A sífilis congênita mostra-se como um evento de alta magnitude e com indicadores desfavoráveis. Assim, é necessário que o novo modelo organizativo da Atenção a Saúde de nosso estado, na oferta de ações e serviços de saúde nos municípios, que compõem as Regiões de Saúde de nosso estado, adotem novas estratégias de impelementação de medidas de controle, para que possam efetivamente atingir a meta de 0,5 casos em cada mil nascidos vivos.

Este estudo pode possibilitar que cada Região de Saúde, a partir de um diagnóstico situacional, desenvolvam medidas de controle adequadas para o perfil demográfico, ampliando a oferta de serviços e alcançando os grupos populacionais, em especial, os mais vulneráveis.

Como ponto forte na realização deste estudo foi a disponibilidade dos dados atualizados no TabNET, do Sistema de Informação sobre Mortalidade SIM e do Sistema de Informação de Agravos de Notificação - SINAN, no site da Diretoria de Vigilância Epidemiológica da Secretaria Estadual de Saúde de Santa Catarina. É um estudo pioneiro que permite demonstrar a magnitude da sífilis congênita para possíveis ampliações de estratégias e implementações do Programas Municipais de Controle de Infecções Sexualmente Transmissíveis de nosso estado.

\section{Contribuições das autoras}

Nass SL e Santos VC participaram da concepção ou desenho do estudo/pesquisa, coleta dos dados, busca e análise estatística dos dados da pesquisa, interpretação dos resultados e redação do artigo científico. Schultz LF participou da concepção ou desenho do estudo/pesquisa, interpretação dos resultados e redação do artigo científico. Moreira TMA participou da concepção ou desenho do estudo/pesquisa, interpretação dos resultados, análise estatística dos dados da pesquisa, interpretação dos resultados e redação do artigo científico.

\section{Conflito de interesses}

Nenhum conflito financeiro, legal ou político envolvendo terceiros (governo, empresas e fundações privadas, etc.) foi declarado para nenhum aspecto do trabalho submetido (incluindo, mas não se limitando a subvenções e financiamentos, participação em conselho consultivo, desenho de estudo, preparação de manuscrito, análise estatística, etc.).

\section{Referências}

1. Radolf JD, Deka RK, Anand A, Smajs D, Norgard MV, Yang XF. Treponema pallidum, the syphilis spirochete: making a living as a stealth pathogen. Nat Rev Microbiol. 2016;14(12):744-59. https:// doi.org/10.1038/nrmicro.2016.141

2. Ministério da Saúde (Brasil), Secretaria de Vigilância em Saúde. Vigilância em Saúde no Brasil 2003|2019: da criação da Secretaria de Vigilância em Saúde aos dias atuais. Boletim Epidemiológico [Internet]. Brasília: Ministério da Saúde; 2019 [citado em 2021 jun 19]. Disponível em: https://www.gov.br/saude/pt-br/assuntos/ boletins-epidemiologicos

3. Moline HR, Smith JF. The continuing threat of syphilis in pregnancy. Curr Opin Obstet Gynecol. 2016;28(2):101-4. https:// doi.org/10.1097/gco.0000000000000258

4. Galvis AE, Arrieta A. Congenital Syphilis: A U.S. Perspective. Children. 2020;7(11): 203. https://doi.org/10.3390/ children7110203

5. Kimball A, Miele K, Bachmann L, Thorpe P, Weinstock H, Bowen V. Missed Opportunities for Prevention of Congenital Syphilis. MMWR Morb. Mortal. Wkly. Rep. 2020;69(22):661-5. https:// dx.doi.org/10.15585\%2Fmmwr.mm6922a1

6. World Health Organization. Guidelines for the treatment of Treponema pallidum (syphilis) [Internet]. Geneva: WHO; 2016. [citado em 2021 jun 20]. Disponível em: https://www.who. int/reproductivehealth/publications/rtis/syphilis-treatmentguidelines/en/ 
7. Korenromp EL, Rowley J, Alonso M, Mello MB, Wijesooriya NS, Mahiané SG, et al. Global burden of maternal and congenital syphilis and associated adverse birth outcomes-Estimates for 2016 and progress since 2012. PLOS ONE. 2019;14(2):e0211720. https://doi.org/10.1371/journal.pone.0211720

8. Secretaria de Estado da Saúde de Santa Catarina (Brasil), Diretoria de Vigilância Epidemiológica. Informativo Epidemiológico Barriga Verde. Boletim epidemiológico sífilis em Santa Catarina 2018 [Internet]. Florianópolis: Diretoria de Vigilância Epidemiológica da Secretaria de Estado da Saúde de Santa Catarina; 2019. [citado em 2021 jun 20]. Disponível em: http:// www.dive.sc.gov.br/barrigaverde/pdf/BV Sifilis.pdf

9. Governo do Estado de Santa Catarina (Brasil), Secretaria de Estado da Saúde. Sistema Único de Saúde. Plano diretor de regionalização: PDR. 2012 [Internet]. Florianópolis: IOESC; 2012. [citado em 2021 abr 10]. Disponível em: https://www. saude.sc.gov.br/index.php/documentos/informacoes-gerais/ planejamento-em-saude/instrumentos-de-gestao-estadual/ plano-diretor-de-regionalizacao/8141-plano-diretor-deregionalizacao-2012/file

10. Resolução n. 466, de 12 de dezembro de 2012 (Brasil). Aprova as diretrizes e normas regulamentadoras de pesquisas envolvendo seres humanos e revoga as Resoluções CNS nos. 196/96, 303/2000 e 404/2008. [Internet]. 2012 dez 12. Disponível em: http://conselho.saude.gov.br/resolucoes/2012/Reso466.pdf

11. Silva IMD, Leal EMM, Pacheco HF, Souza Júnior JG, Silva FS. Perfil epidemiológico da sífilis congênita. Rev enferm UFPE online [Internet]. 2019;13(3):604-13. Disponível em: https://periodicos. ufpe.br/revistas/revistaenfermagem/article/view/236252/31536

12. Maschio-Lima T, Machado ILL, Siqueira JPZ, Almeida MTG. Perfil epidemiológico de pacientes com sífilis congênita e gestacional em um município do Estado de São Paulo, Brasil. Rev. Bras. Mater. Infant. 2019;19(4):865-72. https://doi. org/10.1590/1806-93042019000400007

13. Cavalcante PAM, Pereira RBL, Castro JGD. Sífilis gestacional e congênita em Palmas, Tocantins, 2007-2014. Epidemiol. Serv. Saúde. 2017;26(2):255-64. https://doi.org/10.5123/S167949742017000200003

14. Ministério da Saúde (Brasil), Secretaria de Atenção à Saúde, Departamento de Atenção Básica. Atenção ao pré-natal de baixo risco [Internet]. Brasília: Editora do Ministério da Saúde; 2012. [citado em 2020 out 18]. Disponível em: https://bvsms.saude.gov. br/bvs/publicacoes/cadernos_atencao_basica_32_prenatal.pdf
15. Lima VC, Mororó RM, Martins MA, Ribeiro SM, Linhares MSC. Perfil epidemiológico dos casos de sífilis congênita em um município de médio porte no nordeste brasileiro. J Health Biol Sci. 2017;5(1):56-61. http://dx.doi.org/10.12662/2317-3076jhbs. v5i1.1012.p56-61.2017

16. Rocha RPS, Terças ACP, Nascimento VF, Silva JH, Gleriano JS. Análise do perfil epidemiológico de sífilis nas gestantes e crianças, em Tangará da Serra, de 2007 a 2014. Rev Norte Mineira Enf [Internet]. 2016;5(2):03-21. Disponível em: https://www.periodicos. unimontes.br/index.php/renome/article/view/1246/1295

17. Pan American Health Organization (PAHO). Elimination of Mother-to-Child Transmission of HIV and Syphilis in the Americas - Update 2015 [Internet]. Washington, DC: PAHO; 2015. [citado em 2020 out 25]. Disponível em: https://iris.paho.org/ handle/10665.2/18372

18. Rego AS, Costa LC, Rodrigues LS, Garcia RAS, Silva FMAM, D'eça Junior, et al. Congenital syphilis in Brazil: distribution of cases notified from 2009 to 2016. Rev Soc Bras Med Trop. 2020;53:(e20200338). https://doi.org/10.1590/0037-8682-03382020

19. Mesquita KO, Lima GK, Filgueira AA, Flôr SMC, Freitas CASL, Linhares MSC, et al. Análise dos casos de sífilis congênita em Sobral, Ceará: contribuições para assistência pré-natal. DST-J Bras Doenças Sex Transm. 2012;24(1):20-7. http://dx.doi. org/10.5533/2177-8264-201224107

20. Saraceni V, Pereira GFM, Silveira MF, Araujo MAL, Miranda AE. Vigilância epidemiológica da transmissão vertical da sífilis: dados de seis unidades federativas no Brasil. Rev Panam Salud Publica. 2017;41:e44. https://dx.doi.org/10.26633\%2FRPSP.2017.44

21. Bezerra MLMB, Fernandes FECV, Nunes JPO, Baltar SLSMA, Randau KP. Congenital Syphilis as a Measure of Maternal and Child Healthcare, Brazil. Emerg Infect Dis. 2019;25(8):1469-76. https://doi.org/10.3201/eid2508.180298

22. Costa CV, Santos IAB, Silva JM, Barcelos TF, Guerra HS. Sífilis congênita: repercussões e desafios. Arq. Catarin Med [Internet]. 2017;46(3):194-202. Disponível em: https://pesquisa.bvsalud.org/ portal/resource/pt/biblio-849511

23. Domingues RMSM, Leal MC. Incidência de sífilis congênita e fatores associados à transmissão vertical da sífilis: dados do estudo Nascer no Brasil. Cad. Saúde Pública. 2016;32(6):e0008241. https://doi.org/10.1590/0102-311X00082415

24. Lazarini FM, Barbosa, DA. Intervenção educacional na Atenção Básica para prevenção da sífilis congênita. Rev LatinoAm Enfermagem. 2017;25:e2845. https://doi.org/10.1590/15188345.1612 .2845 